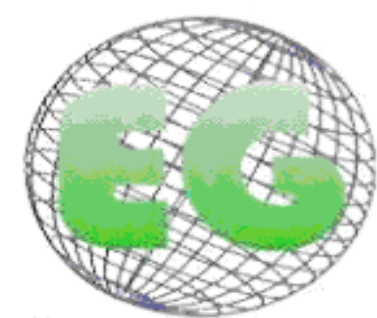

ISSN 1695-6141 $\mathrm{N}^{\circ} 22$

\title{
ENFERMERÍA, MALTRATO DE GÉNERO Y PRESENCIA DE MENORES: REDESCUBRIENDO NUESTRO PAPEL
}

NURSING, GENDER VIOLENCE AND EFFECTS ON CHILDREN: REDEFINING OUR ROLE

\author{
${ }^{*}$ Cabrera Espinosa, M., ** Granero Alted, MJ. \\ *Doctor por la Universidad de Granada. Enfermero de la Unidad de Agudos de Salud Mental. **Diplomada en \\ Ciencias Jurídicas. Enfermera de Urgencias Pediátricas. Complejo Hospitalario de Jaén.
}

Palabras clave: Violencia de género; Enfermería; Maltrato a menores; Salud y género.

Keywords: Gender violence, Nursing, Children abuse, Health and gender.

\section{RESUMEN}

Con esta investigación intentamos comprender el drama familiar por el que atraviesan las hijas e hijos de aquellas parejas en las que se ejerce la violencia de género. En concreto, pretendemos evaluar los efectos que la violencia de género tiene sobre los hijos de las parejas, comprender el modo en que estos efectos se producen, y revalorizar el decisivo papel que el sistema de salud, y sus profesionales, están llamados a realizar ante esta problemática social. Para lograr nuestros objetivos hemos realizado un estudio cualitativo no probabilístico, consistente en la realización de entrevistas no estructuradas de carácter retrospectivo a dieciocho hombres, que en el momento de la investigación se encontraban en prisión por condena firme por maltrato de género en las relaciones de pareja. Posteriormente realizamos un análisis de contenido de los discursos generados. En este análisis descubrimos el modo en que la convivencia en un ambiente de maltrato afecta a los menores, tanto en la inmediatez como en su futuro desarrollo, y cómo la enfermería está llamada a desarrollar un papel central en la detección y el tratamiento, como miembro de un equipo multidisciplinar, de este grave problema actual.

\section{ABSTRACT}

The aim of this investigation is to look at the situation of families where gender violence is taking place in terms of its effects on children, focusing especially on assessing and understanding the specific causes of these effects, whilst analysing the role that health systems and nursing staff can play in tackling this social issue. To this aim, we carried out a non-probabilistic qualitative study which consisted of a series of non-structured retrospective interviews with 18 men who were serving a prison sentence for violence against their partners at the time of the investigation. A subsequent analysis of the content of the responses generated helped us to understand the effects that living in an environment where gender violence is taking place has on children, both in their current and future development, as well as to identify the role that nursing staff can play in detecting and responding to this serious problem as part of a multidisciplinary team. 


\section{INTRODUCCIÓN}

La problemática de la violencia de género se ha convertido en un tema de candente actualidad en nuestra sociedad, prueba de ello es la cantidad de políticas públicas que contra la misma se están desarrollando en España en los últimos años, y que han culminado recientemente con la Ley Orgánica 1/2004 de 28 de diciembre, de Medidas de Protección Integral contra la Violencia de Género ${ }^{1}$.

Casi a diario aparecen nuevas publicaciones en referencia a la violencia de género, pero en todo este drama localizamos un actor que permanece olvidado e invisible en la mayor parte de los casos, nos referimos a las hijas e hijos que conviven en un hogar donde se practica este tipo de violencia. En definitiva, hogares donde maltratador y maltratada son su padre y su madre, las personas que más quieren, y aquellas destinadas a educarlos y a introducirlos en la sociedad.

Con esta investigación hemos pretendido, a través de la investigación cualitativa ${ }^{2,3}$, acercarnos a la problemática de estos pequeños actores mudos del drama familiar que supone la presencia de la violencia de género en su hogar.

\section{Descripción de conceptos y variables}

Antes de adentrarnos en la plasmación de la metodología y los resultados obtenidos, nos parece necesario acotar y definir a qué nos referimos cuando hablamos de violencia de género. Ésta puede ser definida como todo acto de violencia basado en la pertenencia al sexo femenino, que tenga o pueda tener como resultado un daño o sufrimiento físico, sexual o psicológico para las mujeres, inclusive las amenazas de tales actos, la coacción o la privación arbitraria de libertad, tanto si se produce en la vida pública o privada ${ }^{4}$.

Nuestro estudio está centrado en la violencia de género que se produce en el ámbito doméstico y específicamente aquella en la que el maltratador es la pareja o ex pareja de la mujer maltratada ${ }^{5}$. Al calificar a este tipo de violencia con el vocablo género, lo que hacemos es aceptar que estamos ante un tipo de violencia que deriva de la desigualdad de poder entre hombres y mujeres. Por tanto, se produce por la existencia de un código patriarcal de valores que pregona la superioridad masculina frente a la inferioridad femenina, todo producto de una deformación y degeneración de valores y actitudes asociadas a la masculinidad, en un proceso donde lo activo se convirtió en intervencionismo, lo combativo degeneró en agresivo, lo que fue seguridad en sí mismo se convirtió en dominación, la fuerza se convirtió en violencia y abuso; el egocentrismo se convirtió en explotación, la mente unidireccional se convirtió en despotismo, el rigor en intransigencia y el anhelo de superación se convirtió en ambición ${ }^{6}$.

Estamos ante una problemática muy amplia, tanto en número de casos, como en la repercusión social asociada a los casos más graves que suelen terminar con la muerte de la mujer $^{7-9}$. En España estas muertes se han estabilizado, alrededor de 70 mujeres anuales. Sin embargo, el número de denuncias aumenta anualmente pasando de las 43.313 en el año 2002 a las 63.347 del año 2007. A pesar de este aumento, hemos de recordar que un aumento de las denuncias por maltrato de género no necesariamente indica un aumento de este tipo de delitos, sólo puede ser una muestra de la emergencia y la salida a la luz del problema ante un entorno socio cultural mucho más favorable a la erradicación de estas situaciones discriminatorias. Si lo que analizamos son las sucesivas macroencuestas realizadas por Sigma Dos ${ }^{7,8,9}$ para el Instituto de la Mujer, en el año 1999 un 12,4\% (1.865.000 mujeres) se pueden considerar mujeres tipo A o mujeres técnicamente 
maltratadas, cifra que en el 2002 sigue siendo elevada, el 11.1\%, y aunque disminuye en el 2006 al $9.6 \%$ de la población femenina, sin embargo todavía suponen 1.786 .978 mujeres.

\section{Consecuencias de la violencia de género en los hijos testigos de la misma}

Toda violencia produce diferentes tipos o modalidades de consecuencias. Para los objetivos de la presente investigación, nuestro interés se centró en la comprensión de las consecuencias que este tipo de violencia produce en los hijos testigos de la misma ${ }^{10}$.

El primer dato significativo que tenemos que destacar es que entre un 30 y un $60 \%$ de los niños que viven en familias donde existe maltrato de género, ellos también son maltratados. Pero es que, como señala la UNICEF, aunque no se les maltrate físicamente, presenciar o escuchar situaciones violentas tiene efectos psicológicos negativos en los hijos. Sin ser el objeto directo de las agresiones, padecen violencia psicológica, que es una forma de maltrato infantil y que la Convención Internacional de los Derechos del Niño -ratificada por España- considera una forma de maltrato infantil y la recoge en el artículo 19 como violencia mental ${ }^{11}$.

Pensemos que, en la mayoría de los casos, la violencia se produce en etapas donde los niños maduran su desarrollo psicológico; alterando enormemente este desarrollo ante la presencia de las agresiones de una figura primordial de referencia en su desarrollo como es el padre, sobre el agente de socialización por excelencia que es la madre. Los hijos e hijas de un maltratador crecen inmersos en el miedo, siendo candidatos al diagnóstico de toda la variedad de trastornos por estrés traumáticos, depresiones por desesperanza o de posibles trastornos de personalidad ${ }^{12}$.

Además de todo lo mencionado anteriormente, se producen efectos a más largo plazo en estos testigos mudos de la violencia familiar. El más destacado es la llamada trasmisión generacional de la violencia de género. Se ha comprobado que estos niños, de mayores, con más frecuencia y probabilidad maltratarán a sus parejas y que las niñas serán víctimas de violencia de género. $Y$ este efecto sobre el futuro desarrollo de los menores se produce por la permanente presencia de unos patrones violentos de comportamiento y relación que se aplicarán a sus propias relaciones, desarrollando conductas sexistas, patriarcales y violentas. En definitiva, el ejercicio de la violencia doméstica siempre afecta a los niños, bien como receptores, bien como testigos ${ }^{13}$.

\section{El sistema de salud frente a la violencia de género}

El sistema de salud, está llamado a convertirse en uno de los pilares fundamentales en la detección y la prevención de los efectos que la violencia de género tiene sobre estos menores ${ }^{11}$. Será a éste donde acudirán, casi como primera opción, tanto la mujer maltratada como los hijos e hijas de estas parejas, presentando una variada y diversa sintomatología producida por la situación de maltrato. Entre la gran variedad de alteraciones tanto psíquicas como somáticas que aparecen en las mujeres maltratadas destacan la ansiedad, angustia, irritabilidad, inapetencia sexual, insomnio, fatiga permanente o dolores de cabeza y espalda, Incluso la incidencia de la gripe está levemente aumentada en las mujeres que sufren maltrato 9 .

Por tanto, los profesionales que más directamente tratan a la mujer y a sus hijos, enfermeras y médicos, son los que deben estar alerta y detectar ciertos síntomas y signos que nos prevengan sobre la posible existencia de maltrato. 
Hecha esta primera aproximación teórica al problema de estudio, pasamos a describir la investigación realizada y los resultados obtenidos.

\section{MATERIAL Y MÉTODO}

Para comprender la influencia de la violencia de género en los hijos de la pareja, hemos realizado un estudio cualitativo no probabilístico, consistente en la realización de entrevistas no estructuradas de carácter retrospectivo, a dieciocho hombres que en el momento de la investigación se encontraban en prisión por condena firme por maltrato de género en las relaciones de pareja. Para la realización de las entrevistas se solicitó autorización al Ministerio del Interior, se informó a los internos de los objetivos de la investigación, y previamente al inicio de los encuentros personales, se recogió por escrito un consentimiento informado por parte de la persona a entrevistar. Así mismo, hemos garantizado la intimidad de los informantes protegida en el artículo 18.1 de la Constitución.

Nos hemos encontrado ante un grupo de hombres bastante heterogéneo, compuesta por 18 personas varones con edades comprendidas entre los 23 y los 70 años, situándose la edad media en 41,2 años, el $72.2 \%$ se encuentra entre los 30 y los 50 años. En cuanto al nivel de estudios, predominan claramente aquellos que sólo han realizado estudios primarios, son doce personas $(66,68 \%)$, sin estudios tenemos a dos entrevistados $(11,11 \%)$, otros dos tienen terminado el bachillerato $(11,11 \%)$, uno posee una diplomatura $(5,55 \%)$ y otro es licenciado (5,55\%). Diecisiete de ellos tienen hijos, con una media de los mismos de 2.3.

Con nuestro grupo de entrevistados hemos logrado la saturación teórica tanto siguiendo los criterios de propósito teórico como el de relevancia ${ }^{14}$.

Una vez realizados los encuentros y transcritas las entrevistas, efectuamos un análisis de contenido de los discursos generados por nuestros informantes ${ }^{15}$, ayudándonos de un programa de análisis cualitativo de datos asistido por ordenador, el Atlas $\mathrm{Ti}^{16}$.

Para comprender los efectos y el modo en que un entorno de violencia de género afecta a los menores que conviven en él, hemos estudiado tres áreas bien diferenciadas en el discurso del maltratador:

- En primer lugar, analizamos las actitudes y sentimientos que el maltratador mantiene hacia sus hijos.

- En segundo lugar, intentamos acceder a un mayor conocimiento del entorno de convivencia y los valores más representativos de este tipo de familias.

- En último lugar, estudiamos la posible trasmisión generacional de la violencia de género a través del relato que el maltratador realiza de su infancia.

\section{RESULTADOS}

Expondremos a continuación los resultados obtenidos en cada una de las áreas exploradas.

\section{Descripción de las actitudes y sentimientos del maltratador hacia sus hijos e hijas}

Es indudable que la presencia de los hijos, los sentimientos hacia ellos o una valoración de su importancia para la estabilidad de la pareja, debería de emerger a lo largo del discurso. 
Además nosotros llevábamos un apartado específico que intentaba valorar el significado y la importancia que los hijos tienen para nuestros informantes.

Lo primero que hay que destacar es que la presencia de los hijos no es un factor utilizado por el maltratador como coadyuvante de la violencia de género. Para su modo de percibir la relación con su pareja, la presencia de los hijos apenas ha interferido en esta relación.

Los entrevistados tampoco muestran una gran alegría ante la llegada de los hijos, esta presencia está tratada con un gran aplanamiento emocional. Hablan poco del significado que los hijos tienen para él y de su desarrollo y sentimientos hacia su rol paternal; las veces que se menciona directamente a éstos, lo es para exponer aspectos negativos de los mismos, relacionados con temas económicos. Así en el 70\% de los casos, los hijos representan un aumento del gasto económico, y esto sucede en unas personas en las que hemos encontrado que el aspecto económico se encuentra en el centro de sus vidas. Al respecto, nos dice un informante:

Inf3:

"A los cuatro años vinieron los niños y todo bien sólo que tuvimos que cambiar porque en vez de mantener a dos mantienes a cuatro. Ese es el gran cambio de los niños, que tienes que repartir el mismo sueldo entre más gente".

Sólo en un caso se reconoce que son los hijos los que sufren en todo este proceso, pero circunscribe este sufrimiento a la lucha judicial por la custodia, no a la presencia de la violencia en el hogar:

Inf1:

"En este problema son los hijos los que sufren y es que existe una lucha en el juzgado por los hijos y de esta forma, con la denuncia el padre desaparece. Los hijos son los verdaderos paganos de este proceso".

En todos los casos analizados, los hijos son utilizados como modo de desvalorizar a la mujer y como objeto a través del cual aumentar el control sobre la misma:

Inf5:

"Hay mujeres que no les dan una buena educación a los hijos y lo que hacen es hacerlos unos delincuentes, luego llega el padre de estar trabajando todo el día y tiene que poner orden en la casa".

Encontramos, en definitiva una utilización de los hijos para controlar a la mujer, una ausencia de cariño hacia los hijos e hijas y un rol centrado más en lo económico que en lo paternal.

\section{Entorno de convivencia y valores más representativos en este tipo de familias}

Otro de los aspectos destacable de la investigación ha consistido en extraer una imagen del entorno de este tipo de familias. Encontramos un sistema de valores y creencias de un hombre que necesita controlar y mantener la unidad familiar, un hombre con un potente concepto de masculinidad patriarcal dominante y en el que lo material, centrado fundamentalmente en el trabajo y el dinero, se impone a lo espiritual. Para justificar su actitud, la excusa argumentada es la presencia de una mujer minusvalorada, culpable de la 
situación que ha ocurrido. Una mujer que tiene abandonadas las obligaciones y funciones "típicas de su género"; una mujer que abandona el cuidado de la casa.

Para apreciar con mayor claridad los valores dominantes, hemos analizado tanto la autopercepción, como la percepción de la otra. Es decir, cómo se definen a ellos mismos y cómo definen a su pareja. En cuanto a la autopercepción, ellos son fundamentalmente: trabajadores, religiosos, buenas personas, ahorradores y humildes.

Gráfico 1: Autodefinición de los entrevistados
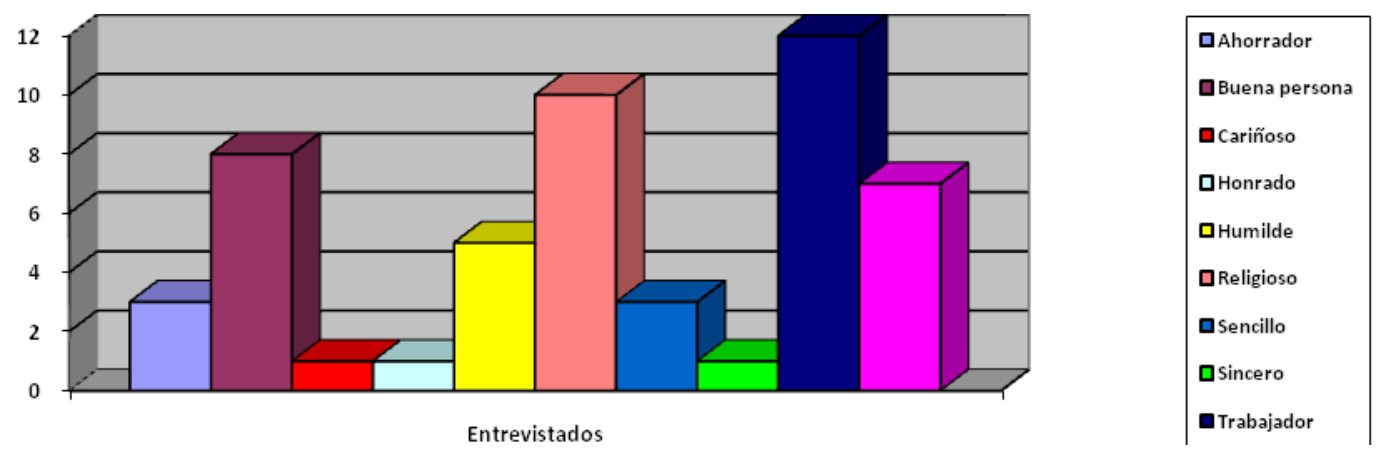

Por el contrario la mujer es: interesada, engañosa, culpable y maltratadora.

Gráfico 2: descripción de la pareja o ex pareja.

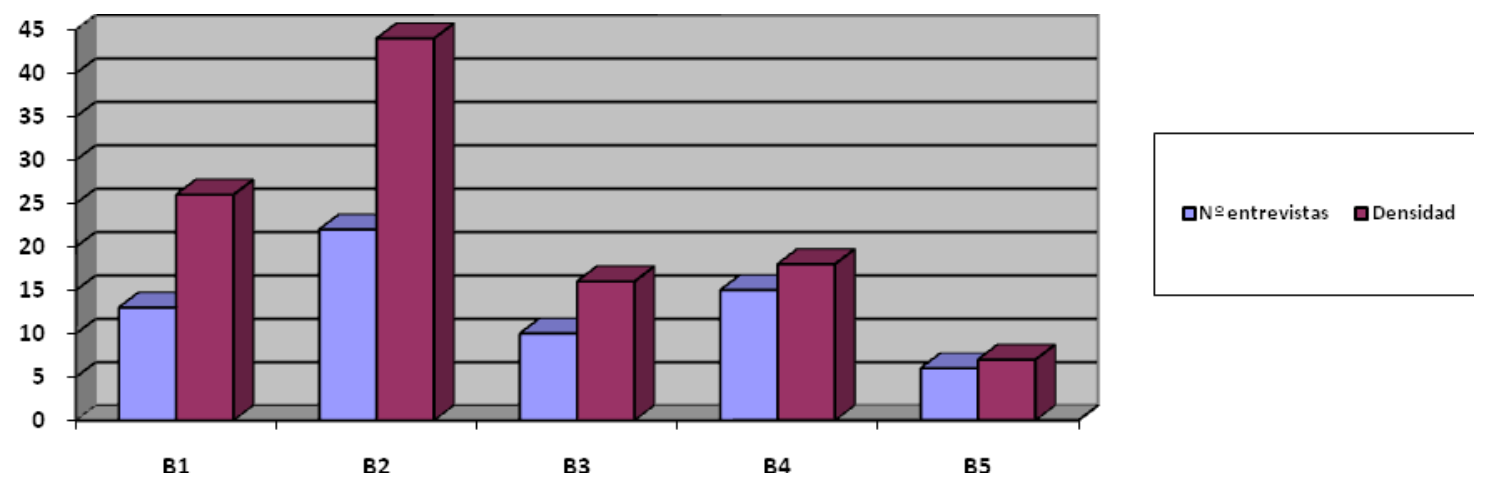

B1: Mujer culpable y maltratadora.

B2: Mujer interesada y engañosa.

B3: Mujer que abandona las labores de la casa y mujer minusvalorada.

B4: Mujer loca, débil y arrepentida.

B5: Mujer con descripciones positivas.

En estos hogares se entienden las relaciones hombre/mujer como sumisión más que como cooperación. Existe un dominio total de la masculinidad sobre la feminidad. Encontramos en el discurso de nuestros informantes una ausencia casi total de vocablos y expresiones hasta 
ahora asociadas a lo femenino; por el contrario, aparecen frecuentemente vocablos asociados con valores masculinizados.

Intentando cuantificar la diferencia de expresiones asociadas a lo masculino en relación con las expresiones asociadas a la feminidad a través de un recuento frecuencial realizado en dos búsquedas:

- En la primera, hemos introducido aquellos conceptos que están más relacionados con la masculinidad, hablamos de vocablos como: trabajador, trabajo, fuerte, dirigir, mandar, mando, esfuerzo, dureza, poder, éxito, lucha, honradez, honrado.

- La segunda de nuestras búsquedas, la hemos realizado con aquellas palabras que más directamente están relacionadas con los conceptos que definen en nuestra sociedad a la feminidad, en concreto: sinceridad, sincera, sensibilidad, comprensión, diálogo, aceptación, aceptar, acatar, felicidad, amor, docilidad, dócil, cariño, llorar, llanto, amabilidad, amable.

Los resultados obtenidos son, en un principio, bastante sorprendentes y abrumadores. En la búsqueda de aquellas palabras relacionadas con la masculinidad nos aparecen 108 entradas, frente a las 6 entradas encontradas en la búsqueda de los vocablos relacionados con la feminidad. Nos encontramos, pues, con unos informantes que manejan un discurso donde la masculinidad es la norma y la feminidad la excepción.

\section{Estudio de la trasmisión generacional de la violencia de género}

Si existe una trasmisión generacional de la violencia de género ésta tendrá que ser rastreada en la infancia de nuestros maltratadores. Por ello buscaremos a través del relato de la infancia de nuestros informantes aquellos rasgos que evidencien la presencia de maltrato de género en sus familias de origen.

Hemos de decir que si lo que buscamos es directamente un relato de maltrato de género en los padres de nuestros entrevistados éste sólo es visible en dos casos (11\%). En otros tres (16\%) han existido episodios de maltrato esporádicos, sin embargo ellos lo justifican y lo asumen como una necesidad y una normalidad. El vocablo que más usan para referirse a su infancia es el de normalidad, e insisten en que ellos no han vivido ni sufrido la violencia de género.

Sin embargo, si en el análisis de contenido aplicamos la perspectiva de género ${ }^{17}$, y por tanto buscamos una definición ampliada de violencia de género que comprenda tanto los aspectos físicos como los psíquicos, así como el ambiente de clara discriminación de género, encontramos datos mucho más interesantes. Así, estamos ante personas que han sido socializadas en ambientes muy masculinizados y competitivos, de hecho en sus familias de origen existe un dominio absoluto de lo masculino sobre lo femenino; y no ya sólo en el aspecto cultural, también en el material, al surgir un dominio de los hijos sobre las hijas. En concreto, si analizamos el sexo dominante en las familias de origen (recuento de hermanos/as por sexo) de nuestros entrevistados, nos encontramos con 53 varones frente a 22 mujeres. Nuestros informantes han tenido una infancia dura, donde el maltrato, definido éste más ampliamente que el puramente físico, ha sido la norma. Son personas en las que se han depositado fuertes expectativas de ascenso en el futuro. Familias donde el aspecto material se impone al sentimental. 
A pesar de lo que decimos, no debemos creer que exista un determinismo cultural que obligue a nadie a usar la violencia para conseguir sus fines; la cultura puede ser descrita como un instrumento de continuidad al servicio de la rutina y el orden social, pero también es la sede de la creatividad, de la invención y de la autocrítica, la cultura también es libertad y no simplemente determinismo ${ }^{18}$.

Al hablar de la trasmisión generacional de la violencia de género nos apoyábamos en las tesis expuestas en multitud de obras, como los trabajos de Dobash ${ }^{19}$, Dutton ${ }^{20}$, Gelles ${ }^{21}$, Okun $^{22}$, Rosenbaum ${ }^{23}$, Walker ${ }^{24}$. No podemos poner en duda las tesis de estos autores; sin embargo, toparnos con unas familias de origen tan masculinizadas y con unos hogares típicamente patriarcales nos llevan a pensar que es la masculinización de estas familias, en definitiva el dominio de una forma de entender y de relacionarse con el mundo mucho más masculina que femenina, lo que favorecerá la elección de la mujer como blanco de la ira del agresor.

\section{CONCLUSIONES}

Encontramos una alta correlación entre una infancia donde ha imperado el maltrato, entendido este en su contexto amplio y no sólo físico, y la presencia de hombres adultos que aceptan la agresividad como modo de resolución de problemas.

Existe, así mismo, una fuerte correlación entre una infancia donde sobresalen los valores de la masculinidad, la hombría o el machismo y la presencia de hombres adultos que defienden a ultranza estos mismos valores. $Y$ es que los hogares de los maltratadores son hogares donde impera la desconfianza, la competitividad, la agresividad y todos los factores asociados a la hombría y la masculinidad. En una gran medida son una réplica de los valores imperantes durante su proceso de socialización familiar en su infancia; y provocará en un futuro consecuencias negativas en los hijos e hijas socializados en ese ambiente familiar, entre las que destacará la trasmisión generacional de la violencia de género.

Los menores que conviven en hogares en donde se ejerce la violencia de género son los pequeños testigos mudos y olvidados de este drama familiar; tanto de las políticas públicas, de los actores sociales, como del discurso del maltratador. Existe una ausencia de programas específicos para la detección y el tratamiento de hijos e hijas de parejas en las que existe violencia de género ${ }^{25}$. Es necesaria la creación de programas que tomen a los menores como sujetos de derechos.

Hay que sacar a la luz, visibilizar la problemática de estos menores, y en esta labor el personal sanitario, y fundamentalmente el de enfermería, tiene una importante labor, por ser el sistema sanitario uno de los primeros recursos a donde acude la mujer que sufre maltrato. Justo por esta razón, es preciso realizar programas específicos de formación, tanto en género como en las consecuencias de la violencia de género sobre el menor, a los profesionales de la salud, enfermeras y médicos fundamentalmente, que son el primer y esencial eslabón en la detección precoz de la violencia de género y sus perversos efectos. Además, el sistema de salud debe de implantar protocolos específicos para detectar no sólo la violencia de género, también la presencia de menores que convivan en hogares víctimas de la misma. Es necesario introducir la perspectiva de género en nuestro quehacer profesional diario, (como ya viene introducida como estrategia en el Plan Integral de Salud Mental de Andalucía 2008-2012 ${ }^{26}$ ). Los profesionales sanitarios debemos evitar la sobre medicación de mujeres y menores maltratados e intervenir activamente para la erradicación de esta desigualdad. 
Las ciencias no están exentas de valores, sus profesionales tampoco. Recordamos, que nosotros como personal de enfermería nos regimos por el Código Deontológico de la Enfermería, donde en su Capítulo VII nos expone el derecho del niño a crecer en salud y dignidad, como obligación ética y responsabilidad social de la enfermería. Y en concreto en su artículo 39 nos recuerda y nos obliga a proteger a los niños de cualquier forma de abuso y a poner en conocimiento de las autoridades competentes cualquier caso del que se tenga conocimiento ${ }^{27}$.

Tampoco somos, las enfermeras y enfermeros, islas desiertas en el océano, debemos interrelacionarnos con el resto de instituciones sociales en un doble sentido: de una parte, estamos obligados a comunicar y cooperar con las instituciones pertinentes en los casos de maltrato, y de otra, los profesionales sanitarios debemos sentirnos y actuar como miembros de la sociedad y no como individuos aislados anclados en la técnica y el diagnóstico.

Debemos comprometernos en ampliar nuestra formación también en aspectos tan importantes y diversos como son el legal o el humanístico, para poder entender y tratar al ser humano no sólo en el aspecto físico, sino también en el mental y en el social. Estamos obligados legal y éticamente a prevenir la aparición de "enfermedades sociales" en nuestros miembros más desprotegidos a través de la búsqueda de una sociedad más justa e igualitaria.

\section{BIBLIOGRAFÍA}

1. BOE número 313, de 29 del 12 del 2004: Ley Orgánica 1/2004, de 28 de diciembre, de Medidas de Protección Integral contra la Violencia de Género. pp. 42166-42197.

2. Alonso, L.E. La mirada cualitativa en sociología. Madrid: Fundamentos, 2003.

3. Bericat, E. La integración de los métodos cuantitativo y cualitativo en la investigación social. Significado y medida. Barcelona: Ariel S.A., 1998.

4. Naciones Unidas: Declaración sobre la Eliminación de la Violencia contra la Mujer.

A/RES/48/104. Ginebra, 1994.

5. Alberdi, I.; Matas, N. La violencia doméstica: informe sobre los malos tratos a mujeres en España. Barcelona: Fundación La Caixa, 2002.

6. Morilla, B. El valor de ser hombre. Historia oculta de la masculinidad. Madrid: Oberan Anaya, 2001.

7. Sigma Dos. I Macroencuesta sobre la violencia contra las mujeres. Madrid: Instituto de la Mujer, 1999.

8. Sigma Dos. II Macroencuesta sobre la violencia contra las mujeres. Madrid: Instituto de la Mujer, 2002.

9. Sigma Dos. III Macroencuesta sobre la violencia contra las mujeres. Madrid: Instituto de la Mujer, 2006.

10. Comisión Europea. Programa Daphne, 2000.

11. Horno, J. (Coord.). Atención a los niños y niñas víctimas de la violencia de género. Análisis de la atención a los hijos e hijas de mujeres víctimas de violencia de género en el sistema de protección a la mujer. Madrid: Save the Children, 2006.

12. Asensi, I. Violencia de género: consecuencias en los hijos. Alicante: Psicojurix, 2003.

13. González, M.E. Género y salud: una reflexión sociológica del caso valenciano. En: Raldf, R. Cuestiones actuales de sociología del género. Madrid: Centro de Investigaciones Sociológicas, 2001, pp. 149-171

14. Jones, D.; Manzelli, H.; Pecheny, M. La teoría fundamentada: su aplicación en una investigación sobre vida cotidiana con VIH/sida y con hepatitis C. En: Kornblit, A.L. (coord.). Metodología cualitativa en ciencias sociales. Buenos Aires: Biblos, 2004, pp. 47-76. 
15. Cartwright, D.P. Análisis del material cualitativo. En: Festinger, L.; Katz, D. Los métodos de investigación en las ciencias sociales. Buenos Aires: Paidós, 1979, pp. 389-432.

16. Muñoz, J. Análisis cualitativo de datos textuales con Atlasti 5. Barcelona: Universidad Autónoma de Barcelona, 2005. Disponible en: http://seneca.uab.es/jmunoz/biblio/Atlas5.pdf. Consultado el 22 de enero del 2008.

17. Puleo, A.H. Filosofía, género y pensamiento crítico. Valladolid: Servicio de publicaciones de la Universidad de Valladolid, 2000.

18. Bauman, Z. La cultura como praxis. Barcelona: Paidós, 2002.

19. Dobash, R. E.; Dobash, R. P. Violence against wives: A case against the patriarchy. Nueva York: Free Press, 1979.

20. Dutton, D. G.; Golant, S. K. El golpeador: un perfil psicológico. Barcelona: Paidós, 1997.

21. Gelles, R. J. The violent home: A study of physical aggression between husbands and wives. Beverly Hills: Sage CA, 1974.

22. Okun, L. Woman abuse: Facts replacing myths. New York: University of New York, 1986.

23. Rosenbaum, A.; O'Leary, K. Marital violence: Characteristics of Abusivecouples. En: Journal of Consulting and Clinical Psychology, n. 49 (1). 1981, pp. 63-71.

24. Walker, L.E. The battered woman syndrome. New York: Springer, 1984.

25. Horno, J. (Coord.). Atención a los niños y niñas víctimas de la violencia de género. Análisis de la atención a los hijos e hijas de mujeres víctimas de violencia de género en el sistema de protección a la mujer. Madrid: Save the Children, 2006.

26. II Plan Integral de Salud Mental de Andalucía. Sevilla: Consejería de Salud, 2008.

27. Código Deontológico de la Enfermería Española, Cap. VII, Art. 39. Disponible en: http://www.cge.enfermundi.com. Consultado el 10 de marzo del 2009. 